\title{
On fourth-order jacobsthal quaternions
}

\author{
Gamaliel Cerda-Morales $^{\mathrm{a}^{*}}$ \\ a Instituto de Matemáticas, Pontificia Universidad Católica de Valparaíso, Blanco Viel 596, Cerro Barón, Valparaíso, Chile. \\ *Corresponding author E-mail: gamaliel.cerda@usm.cl
}

\section{Article Info}

Keywords: Fourth-order Jacobsthal number, Jacobsthal number, quaternion, Recurrence relation

2010 AMS: 11B39, 11R52, 20G20

Received: 18 June 2018

Accepted: 2 August 2018

Available online: 30 September 2018

\begin{abstract}
In this paper, we present for the first time a sequence of quaternions of order 4 that we will call the fourth-order Jacobsthal and the fourth-order Jacobsthal-Lucas quaternions. In particular, we are interested in the generating function, Binet formula, explicit formula and some interesting results for fourth-order Jacobsthal quaternions and fourth-order JacobsthalLucas quaternions. This generalizes some previous results given by Szynal-Liana and Włoch in [13], Torunbalci Aydin and Yüce in [14] and Cerda-Morales in [2].
\end{abstract}

\section{Introduction}

The Jacobsthal numbers have many interesting properties and applications in many fields of science (see, e.g. [1, 4, 12, 9]). In [1], Barry investigated a Jacobsthal decomposition of Pascal's triangle. In [4], Deveci et. al. defined the generalized order- $k$ Jacobsthal sequences modulo $m$. In [12], Köken and Bozkurt showed that the Jacobsthal numbers are also generated by a special matrix. The Jacobsthal numbers $J_{n}$ are defined [9] by the recurrence relation

$$
J_{0}=0, J_{1}=1, J_{n+2}=J_{n+1}+2 J_{n}, n \geq 0 .
$$

Another important sequence is the Jacobsthal-Lucas sequence. This sequence is defined by the recurrence relation

$$
j_{0}=2, j_{1}=1, j_{n+2}=j_{n+1}+2 j_{n}, n \geq 0 .
$$

In [3] the Jacobsthal recurrence relation is extended to higher order recurrence relations and the basic list of identities provided by Horadam [9] is expanded and extended to several identities for some of the higher order cases. Furthermore, the authors generalized the Jacobsthal recursion as

$$
J_{n+r}^{(r)}=\sum_{s=1}^{r-1} J_{n+r-s}^{(r)}+2 J_{n}^{(r)}
$$

with $n \geq 0$ and initial conditions $J_{0}=0$ and $J_{s}=1$ for $s=1, \ldots, r-1$. For the $n$-th order- $r$ Jacobsthal-Lucas numbers $j_{n}^{(r)}$ we use the same recursion with initial conditions $j_{s}^{(r)}=j_{s}^{(r-1)}$ for $s=1, \ldots, r-1$.

In this work we consider the particular case $r=4$, the fourth-order Jacobsthal numbers $\left\{J_{n}^{(4)}\right\}_{n \geq 0}$ and the fourth-order Jacobsthal-Lucas numbers $\left\{j_{n}^{(4)}\right\}_{n \geq 0}$ are defined by

$$
J_{n+4}^{(4)}=J_{n+3}^{(4)}+J_{n+2}^{(4)}+J_{n+1}^{(4)}+2 J_{n}^{(4)}, J_{0}^{(4)}=0, J_{1}^{(4)}=J_{2}^{(4)}=J_{3}^{(4)}=1
$$

and

$$
j_{n+4}^{(4)}=j_{n+3}^{(4)}+j_{n+2}^{(4)}+j_{n+1}^{(4)}+2 j_{n}^{(4)}, j_{0}^{(4)}=2, j_{1}^{(4)}=1, j_{2}^{(4)}=5, j_{3}^{(4)}=10
$$

respectively. 
The first fourth-order Jacobsthal numbers and fourth-order Jacobsthal-Lucas numbers are presented in the following table.

\begin{tabular}{l|llllllllllllll}
\hline$s$ & 0 & 1 & 2 & 3 & 4 & 5 & 6 & 7 & 8 & 9 & 10 & 11 & 12 & $\ldots$ \\
\hline$J_{s}^{(4)}$ & 0 & 1 & 1 & 1 & 3 & 7 & 13 & 25 & 51 & 103 & 205 & 409 & 819 & $\ldots$ \\
$j_{s}^{(4)}$ & 2 & 1 & 5 & 10 & 20 & 37 & 77 & 154 & 308 & 613 & 1229 & 2458 & 4916 & $\ldots$ \\
\hline
\end{tabular}

On the other hand, Horadam [7] introduced the $n$-th Fibonacci and the $n$-th Lucas quaternion as follows

$$
Q_{n}=F_{n}+i F_{n+1}+j F_{n+2}+k F_{n+3}
$$

and

$$
Q_{n}=L_{n}+i L_{n+1}+j L_{n+2}+k L_{n+3},
$$

respectively. Here $F_{n}$ and $L_{n}$ are the $n$-th Fibonacci and $n$-th Lucas numbers, respectively. Furthermore, the basis $i, j, k$ satisface the following rules:

$$
i^{2}=j^{2}=k^{2}=-1, i j k=-1 .
$$

Furthermore, the rules (1.8) imply $i j=-j i=k, j k=-k j=i$ and $k i=-i k=j$. In general, a quaternion is a hyper-complex number and is defined by $Q=q_{r}+i q_{i}+j q_{j}+k q_{k}$, where $i, j, k$ are as in (1.8) and $\left\{q_{r}, q_{i}, q_{j}, q_{k}\right\} \subset \mathbb{R}$. Note that we can write $Q=q_{r}+V_{Q}$ where $V_{Q}=i q_{i}+j q_{j}+k q_{k}$. The conjugate of the quaternion $Q$ is denoted by $\bar{Q}=q_{r}-V_{Q}$. The norm of a quaternion $Q$ is defined by $N r(Q)=Q \bar{Q}=q_{r}^{2}+q_{i}^{2}+q_{j}^{2}+q_{k}^{2} \in \mathbb{R}$.

Many interesting properties of Fibonacci and Lucas quaternions can be found in [5, 6, 7, 8, 10]. In [6], Halici investigated complex Fibonacci quaternions. In [8] Horadam mentioned the possibility of introducing Pell quaternions and generalized Pell quaternions. In [13], the authors defined the Jacobsthal quaternions and the Jacobsthal-Lucas quaternions. Recently, in [2] the author defined the third-order Jacobsthal quaternions and mentioned the possibility of introducing higher order Jacobsthal quaternions.

In this paper, we introduce and study the fourth-order Jacobsthal quaternions and the fourth-order Jacobsthal-Lucas quaternions. In particular, we give generating function, Binet formula and some interesting results for the fourth-order Jacobsthal quaternions and fourth-order Jacobsthal-Lucas quaternions.

For fourth-order Jacobsthal and fourth-order Jacobsthal-Lucas numbers some identities are given, see [3]. In this paper we need some of them.

$$
\begin{aligned}
& j_{n}^{(4)}-6 J_{n}^{(4)}=\left\{\begin{array}{ccc}
2 & \text { if } & n \equiv 0(\bmod 4) \\
-5 & \text { if } & n \equiv 1(\bmod 4) \\
-1 & \text { if } & n \equiv 2(\bmod 4) \\
4 & \text { if } & n \equiv 3(\bmod 4)
\end{array}\right. \\
& 6 J_{n}^{(4)}+j_{n}^{(4)}-j_{n+1}^{(4)}=\left\{\begin{array}{clc}
1 & \text { if } & n \equiv 0,2(\bmod 4) \\
2 & \text { if } & n \equiv 1(\bmod 4) \\
-4 & \text { if } & n \equiv 3(\bmod 4)
\end{array},\right. \\
& J_{n+2}^{(4)}-J_{n}^{(4)}-j_{n-1}^{(4)}=\left\{\begin{array}{clc}
0 & \text { if } & n \equiv 0(\bmod 4) \\
-2 & \text { if } & n \equiv 1(\bmod 4) \\
1 & \text { if } & n \equiv 2,3(\bmod 4)
\end{array} \quad(n \geq 1)\right. \\
& \sum_{s=0}^{n} J_{s}^{(4)}=\left\{\begin{array}{ccc}
J_{n+1}^{(4)}-1 & \text { if } & n \equiv 0(\bmod 4) \\
J_{n+1}^{(4)} & \text { if } & n \equiv 1,3(\bmod 4) \\
J_{n+1}^{(4)}+1 & \text { if } & n \equiv 2(\bmod 4)
\end{array}\right.
\end{aligned}
$$

and

$$
\sum_{s=0}^{n} j_{s}^{(4)}=\left\{\begin{array}{lll}
j_{n+1}^{(4)}-2 & \text { if } & n \neq 0(\bmod 3) \\
j_{n+1}^{(4)}+1 & \text { if } & n \equiv 0(\bmod 3)
\end{array} .\right.
$$

Using standard techniques for solving recurrence relations, the auxiliary equation, and its roots are given by

$$
x^{4}-x^{3}-x^{2}-x-2=0 ; x=2, x=-1, \text { and } x= \pm i .
$$

Note that the latter two are the complex conjugate quartic roots of unity. Call them $\omega_{1}$ and $\omega_{2}$, respectively. Thus the Binet formulas can be written as

$$
J_{n}^{(4)}=\frac{1}{5}\left(2^{n}-\left(\frac{1+3 i}{2}\right) \omega_{1}^{n}-\left(\frac{1-3 i}{2}\right) \omega_{2}^{n}\right)
$$

and

$$
j_{n}^{(4)}=\frac{3}{10}\left(2^{n+2}+\frac{5}{3}(-1)^{n}+\left(\frac{1+3 i}{2}\right) \omega_{1}^{n}+\left(\frac{1-3 i}{2}\right) \omega_{2}^{n}\right)
$$


respectively.

Now, we use the notation

$$
H_{n}^{(4)}(a, b)=\frac{A \omega_{1}^{n}-B \omega_{2}^{n}}{\omega_{1}-\omega_{2}}=\left\{\begin{array}{ccc}
a & \text { if } & n \equiv 0(\bmod 4) \\
b & \text { if } & n \equiv 1(\bmod 4) \\
-a & \text { if } & n \equiv 2(\bmod 4) \\
-b & \text { if } & n \equiv 3(\bmod 4)
\end{array},\right.
$$

where $A=b-a \omega_{2}$ and $B=b-a \omega_{1}$, in which $\omega_{1}$ and $\omega_{2}$ are the complex conjugate quartic roots of unity (i.e. $\omega_{1}^{4}=\omega_{2}^{4}=1$ ). Furthermore, note that for all $n \geq 0$ we have

$$
H_{n+2}^{(4)}(a, b)=-H_{n}^{(4)}(a, b)
$$

where $H_{0}^{(4)}(a, b)=a$ and $H_{1}^{(4)}(a, b)=b$.

From the Binet formulas (1.14), (1.15) and Eq. (1.16), we have

$$
\begin{aligned}
J_{n}^{(4)} & =\frac{1}{5}\left(2^{n}-V_{n}^{(4)}\right), \\
j_{n}^{(4)} & =\frac{3}{10}\left(2^{n+2}+\frac{5}{3}(-1)^{n}+V_{n}^{(4)}\right),
\end{aligned}
$$

where $V_{n}^{(4)}=H_{n}^{(4)}(1,-3)$.

\section{The fourth-order jacobsthal quaternions}

The $n$-th fourth-order Jacobsthal quaternion $J Q_{n}^{(4)}$ and the $n$-th fourth-order Jacobsthal-Lucas quaternion $j Q_{n}^{(4)}$ can be defined as

$$
J Q_{n}^{(4)}=J_{n}^{(4)}+i J_{n+1}^{(4)}+j J_{n+2}^{(4)}+k J_{n+3}^{(4)}
$$

and

$$
j Q_{n}^{(4)}=j_{n}^{(4)}+i j_{n+1}^{(4)}+j j_{n+2}^{(4)}+k j_{n+3}^{(4)}, n \geq 0,
$$

respectively. Here $J_{n}^{(4)}$ and $j_{n}^{(4)}$ are the $n$-th fourth-order Jacobsthal and $n$-th fourth-order Jacobsthal-Lucas numbers, respectively. Furthermore, the basis $i, j, k$ satisface the rules in (1.8).

The function $G(t)=\sum_{n \geq 0} J Q_{n}^{(4)} t^{n}$ is called the generating function for the sequence $\left\{J Q_{n}^{(4)}\right\}$. In [3], the authors found a generating function for fourth-order Jacobsthal numbers. In the following theorem, we established the generating function for fourth-order Jacobsthal and fourth-order Jacobsthal-Lucas quaternions.

Theorem 2.1. The generating function for fourth-order Jacobsthal-Lucas quaternion is

$$
\sum_{n \geq 0} j Q_{n}^{(4)} t^{n}=\frac{\left\{\begin{array}{c}
2+i+5 j+10 k+t(-1+4 i+5 j+10 k)+t^{2}(2+4 i+5 j+7 k) \\
+t^{3}(2+4 i+2 j+10 k)
\end{array}\right\}}{1-t-t^{2}-t^{3}-2 t^{4}} .
$$

Proof. Assuming that the generating function of the quaternion $\left\{j Q_{n}^{(4)}\right\}_{n \geq 0}$ has the form $G(t)=\sum_{n \geq 0} j Q_{n}^{(4)} t^{n}$, we obtain that

$$
\begin{aligned}
\left(1-t-t^{2}-t^{3}-2 t^{4}\right) G(t) & =\left(j Q_{0}^{(4)}+j Q_{1}^{(4)} t+\cdots\right)-\left(j Q_{0}^{(4)} t+j Q_{1}^{(4)} t^{2}+\cdots\right)-\cdots \\
& =j Q_{0}^{(4)}+t\left(j Q_{1}^{(4)}-j Q_{0}^{(4)}\right)+t^{2}\left(j Q_{2}^{(4)}-j Q_{1}^{(4)}-j Q_{0}^{(4)}\right)+t^{3}\left(j Q_{3}^{(4)}-j Q_{2}^{(4)}-j Q_{1}^{(4)}-j Q_{0}^{(4)}\right),
\end{aligned}
$$

since $j Q_{n+4}^{(4)}=j Q_{n+3}^{(4)}+j Q_{n+2}^{(4)}+j Q_{n+1}^{(4)}+2 j Q_{n}^{(4)}(n \geq 0)$ and the coefficients of $t^{n}$ for $n \geq 4$ are equal to zero. In equivalent form is

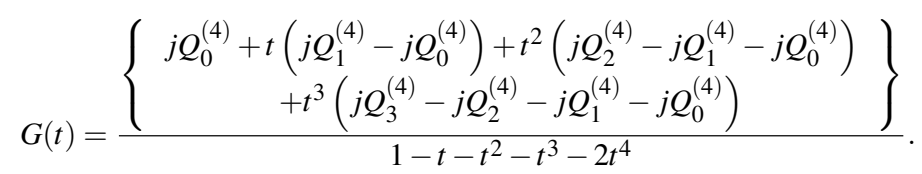

Thus, the proof is completed.

Thus, the Binet formula for $j Q_{n}^{(4)}$ can be given in the following theorem.

Theorem 2.2. If $j Q_{n}^{(4)}=j_{n}^{(4)}+i j_{n+1}^{(4)}+j j_{n+2}^{(4)}+k j_{n+3}^{(4)}$ be the $n$-th fourth-order Jacobsthal-Lucas quaternion. Then,

$$
\begin{aligned}
j Q_{n}^{(4)} & =\frac{3}{10}\left[2^{n+2} \alpha+\frac{5}{3}(-1)^{n} \beta+\left(\frac{1+3 i}{2}\right) \omega_{1}^{n} \underline{\omega_{1}}+\left(\frac{1-3 i}{2}\right) \omega_{2}^{n} \underline{\omega_{2}}\right] \\
& =\frac{3}{10}\left[2^{n+2} \alpha+\frac{5}{3}(-1)^{n} \beta+V Q_{n}^{(4)}\right],
\end{aligned}
$$

where $\omega_{1}$, $\omega_{2}$ are the complex conjugate quartic roots of unity. Furthermore, $\alpha=1+2 i+4 j+8 k, \beta=1-i+j-k$ and $V Q_{n}^{(4)}=$ $V_{n}^{(4)}+i V_{n+1}^{(4)}+j V_{n+2}^{(4)}+k V_{n+3}^{(4)}$. 
Proof. Let $V_{n}^{(4)}=H_{n}^{(4)}(1,-3)$. Using the relation (1.18), we have

$$
\begin{aligned}
\frac{10}{3} \cdot j Q_{n}^{(4)} & =\frac{10}{3}\left(j_{n}^{(4)}+i j_{n+1}^{(4)}+j j_{n+2}^{(4)}+k j_{n+3}^{(4)}\right) \\
& =\left(2^{n+2}+\frac{5}{3}(-1)^{n}+V_{n}^{(4)}\right)+i\left(2^{n+3}-\frac{5}{3}(-1)^{n}+V_{n+1}^{(4)}\right)+j\left(2^{n+4}+\frac{5}{3}(-1)^{n}+V_{n+2}^{(4)}\right)+k\left(2^{n+5}-\frac{5}{3}(-1)^{n}+V_{n+3}^{(4)}\right) \\
& =2^{n+2}(1+2 i+4 j+8 k)+\frac{5}{3}(-1)^{n}(1-i+j-k)+V Q_{n}^{(4)},
\end{aligned}
$$

where $V Q_{n}^{(4)}=V_{n}^{(4)}+i V_{n+1}^{(4)}+j V_{n+2}^{(4)}+k V_{n+3}^{(4)}$. Furthermore,

$$
\begin{aligned}
V Q_{n}^{(4)} & =\left(\left(\frac{1+3 i}{2}\right) \omega_{1}^{n}+\left(\frac{1-3 i}{2}\right) \omega_{2}^{n}\right)+i\left(\left(\frac{1+3 i}{2}\right) \omega_{1}^{n+1}+\left(\frac{1-3 i}{2}\right) \omega_{2}^{n+1}\right) \\
& +j\left(\left(\frac{1+3 i}{2}\right) \omega_{1}^{n+2}+\left(\frac{1-3 i}{2}\right) \omega_{2}^{n+2}\right)+k\left(\left(\frac{1+3 i}{2}\right) \omega_{1}^{n+3}+\left(\frac{1-3 i}{2}\right) \omega_{2}^{n+3}\right) \\
& =\left(\frac{1+3 i}{2}\right) \omega_{1}^{n} \underline{\omega_{1}}+\left(\frac{1-3 i}{2}\right) \omega_{2}^{n} \underline{\omega_{2}},
\end{aligned}
$$

with $\underline{\omega_{1}}=1+\omega_{1} i-j-\omega_{1} k$ and $\underline{\omega_{2}}=1+\omega_{2} i-j-\omega_{2} k$, since $\omega_{1}^{2}=\omega_{2}^{2}=-1$. So, the theorem is proved.

In a similar way, using the Eqs. (2.3) and (2.4) one can easily prove the following theorem.

Theorem 2.3. If $J Q_{n}^{(4)}=J_{n}^{(4)}+i J_{n+1}^{(4)}+j J_{n+2}^{(4)}+k J_{n+3}^{(4)}$ be the $n$-th fourth-order Jacobsthal quaternion. Then,

$$
\begin{gathered}
\sum_{n \geq 0} J Q_{n}^{(4)} t^{n}=\frac{\left(\begin{array}{c}
i+j+k+t(1+2 k) \\
+t^{2}(-i+j+3 k)+t^{3}(-1+2 j+2 k)
\end{array}\right)}{1-t-t^{2}-t^{3}-2 t^{4}}, \\
J Q_{n}^{(4)}=\frac{1}{5}\left[2^{n} \alpha-V Q_{n}^{(4)}\right]
\end{gathered}
$$

where $\alpha=1+2 i+4 j+8 k$ and $V Q_{n}^{(4)}=V_{n}^{(4)}+i V_{n+1}^{(4)}+j V_{n+2}^{(4)}+k V_{n+3}^{(4)}$.

\section{Some identities for the fourth-order jacobsthal quaternions}

By some elementary calculations we find the following recurrence relations for the fourth-order Jacobsthal and fourth-order Jacobsthal-Lucas quaternions from (2.1) and (2.2):

$$
\begin{aligned}
J Q_{n+2}^{(4)}+J Q_{n+1}^{(4)}+ & J Q_{n}^{(4)}+2 J Q_{n-1}^{(4)} \\
= & \left(J_{n+2}^{(4)}+i J_{n+3}^{(4)}+j J_{n+4}^{(4)}+k J_{n+5}^{(4)}\right)+\left(J_{n+1}^{(4)}+i J_{n+2}^{(4)}+j J_{n+3}^{(4)}+k J_{n+4}^{(4)}\right) \\
& +\left(J_{n}^{(4)}+i J_{n+1}^{(4)}+j J_{n+2}^{(4)}+k J_{n+3}^{(4)}\right)+2\left(J_{n-1}^{(4)}+i J_{n}^{(4)}+j J_{n+1}^{(4)}+k J_{n+2}^{(4)}\right) \\
= & \left(J_{n+2}^{(4)}+J_{n+1}^{(4)}+J_{n}^{(4)}+2 J_{n-1}^{(4)}\right)+\left(J_{n+3}^{(4)}+J_{n+2}^{(4)}+J_{n+1}^{(4)}+2 J_{n}^{(4)}\right) i \\
& +\left(J_{n+4}^{(4)}+J_{n+3}^{(4)}+J_{n+2}^{(4)}+2 J_{n+1}^{(4)}\right) j+2\left(J_{n+5}^{(4)}+J_{n+4}^{(4)}+J_{n+3}^{(4)}+2 J_{n+2}^{(4)}\right) k \\
= & J_{n+3}^{(4)}+i J_{n+4}^{(4)}+j J_{n+5}^{(4)}+k J_{n+6}^{(4)} \\
= & J Q_{n+3}^{(4)}
\end{aligned}
$$

and similarly $j Q_{n+3}^{(4)}=j Q_{n+2}^{(4)}+j Q_{n+1}^{(4)}+j Q_{n}^{(4)}+2 j Q_{n-1}^{(4)}$, for $n \geq 1$.

Now, we give some interesting results for the fourth-order Jacobsthal quaternions $\left\{J Q_{n}^{(4)}\right\}_{n \geq 0}$ and the fourth-order Jacobsthal-Lucas quaternions $\left\{j Q_{n}^{(4)}\right\}_{n \geq 0}$.

Theorem 3.1. Let $n \geq 0$ integer. Then, we have

$$
j Q_{n}^{(4)}-6 J Q_{n}^{(4)}=\left\{\begin{array}{cll}
2-5 i-j+4 k & \text { if } & n \equiv 0(\bmod 4) \\
-5-i+4 j+2 k & \text { if } & n \equiv 1(\bmod 4) \\
-1+4 i+2 j-5 k & \text { if } & n \equiv 2(\bmod 4) \\
4+2 i-5 j-k & \text { if } & n \equiv 3(\bmod 4)
\end{array}\right.
$$

Proof. To prove Eq. (3.2) we need the Eq. (1.9). In fact, it suffices to take the Binet's formula of $J_{n}^{(4)}$ and $j_{n}^{(4)}$ in (1.18). Then,

$$
\begin{aligned}
j_{n}^{(4)}-6 J_{n}^{(4)} & =\frac{3}{10}\left(2^{n+2}+\frac{5}{3}(-1)^{n}+V_{n}^{(4)}\right)-\frac{6}{5}\left(2^{n}-V_{n}^{(4)}\right) \\
& =\frac{1}{2}\left((-1)^{n}+3 V_{n}^{(4)}\right) .
\end{aligned}
$$


For definitions (2.1) and (2.2), we have $J Q_{n}^{(4)}=J_{n}^{(4)}+i J_{n+1}^{(4)}+j J_{n+2}^{(4)}+k J_{n+3}^{(4)}$ and $j Q_{n}^{(4)}=j_{n}^{(4)}+i j_{n+1}^{(4)}+j j_{n+2}^{(4)}+k j_{n+3}^{(4)}$. Then, if we consider $n \equiv 0(\bmod 4)$, we obtain

$$
\begin{aligned}
j Q_{n}^{(4)}-6 J Q_{n}^{(4)} & =\left(j_{n}^{(4)}+i j_{n+1}^{(4)}+j j_{n+2}^{(4)}+k j_{n+3}^{(4)}\right)-6\left(J_{n}^{(4)}+i J_{n+1}^{(4)}+j J_{n+2}^{(4)}+k J_{n+3}^{(4)}\right) \\
& =\left(j_{n}^{(4)}-6 J_{n}^{(4)}\right)+i\left(j_{n+1}^{(4)}-6 J_{n+1}^{(4)}\right)+j\left(j_{n+2}^{(4)}-6 J_{n+2}^{(4)}\right)+k\left(j_{n+3}^{(4)}-6 J_{n+3}^{(4)}\right) \\
& =2-5 i-j+4 k
\end{aligned}
$$

since $j_{n+1}^{(4)}-6 J_{n+1}^{(4)}=-5, j_{n+2}^{(4)}-6 J_{n+2}^{(4)}=-1$ and $j_{n+3}^{(4)}-6 J_{n+3}^{(4)}=4$. The other identities are clear from equations (1.9) and (1.18).

Theorem 3.2. Let $n \geq 0$ integer. Then,

$$
N r\left(J Q_{n}^{(4)}\right)=\left\{\begin{array}{lll}
\frac{1}{5}\left(17 \cdot 2^{2 n}-6 \cdot 2^{n}+4\right) & \text { if } & n \equiv 0,1(\bmod 4) \\
\frac{1}{5}\left(17 \cdot 2^{2 n}+6 \cdot 2^{n}+4\right) & \text { if } & n \equiv 2,3(\bmod 4)
\end{array} .\right.
$$

Proof. To prove Eq. (3.3), we use definition of norm for the fourth-order Jacobsthal quaternion $J Q_{n}^{(4)}$,

$$
N r\left(J Q_{n}^{(4)}\right)=\left(J_{n}^{(4)}\right)^{2}+\left(J_{n+1}^{(4)}\right)^{2}+\left(J_{n+2}^{(4)}\right)^{2}+\left(J_{n+3}^{(4)}\right)^{2}
$$

Then, by the Binet formula (1.18) we have

$$
\begin{aligned}
N r\left(J Q_{n}^{(4)}\right) & =\frac{1}{25}\left(\begin{array}{c}
\left(2^{n}-V_{n}^{(4)}\right)^{2}+\left(2^{n+1}-V_{n+1}^{(4)}\right)^{2} \\
+\left(2^{n+2}-V_{n+2}^{(4)}\right)^{2}+\left(2^{n+3}-V_{n+3}^{(4)}\right)^{2}
\end{array}\right) \\
& =\frac{1}{25}\left(\begin{array}{c}
85 \cdot 2^{2 n}-2^{n+1}\left(V_{n}^{(4)}+2 V_{n+1}^{(4)}+4 V_{n+2}^{(4)}+8 V_{n+3}^{(4)}\right) \\
+\left(V_{n}^{(4)}\right)^{2}+\left(V_{n+1}^{(4)}\right)^{2}+\left(V_{n+2}^{(4)}\right)^{2}+\left(V_{n+3}^{(4)}\right)^{2}
\end{array}\right) \\
& =\frac{1}{25}\left(85 \cdot 2^{2 n}+3 \cdot 2^{n+1}\left(V_{n}^{(4)}+2 V_{n+1}^{(4)}\right)+20\right) \\
& =\frac{1}{5}\left(17 \cdot 2^{2 n}+3 \cdot 2^{n+1} U_{n+1}^{(4)}+4\right)
\end{aligned}
$$

where $U_{n}^{(4)}=H_{n}^{(4)}(1,-1)$. Then, if $n \equiv 0,1(\bmod 4)$, we obtain $U_{n+1}^{(4)}=-1$ and $N r\left(J Q_{n}^{(4)}\right)=\frac{1}{5}\left(17 \cdot 2^{2 n}-3 \cdot 2^{n+1}+4\right)$. The other identities are clear from equations (3.4) and (1.16).

In a similar way, using the Eqs. (1.10) and (1.11) one can easily prove the following theorem.

Theorem 3.3. Let $n \geq 0$ integer. Then,

$$
\begin{gathered}
6 J Q_{n}^{(4)}-j Q_{n}^{(4)}-j Q_{n+1}^{(4)}=\left\{\begin{array}{cl}
1+2 i+j-4 k & \text { if } n \equiv 0(\bmod 4) \\
2+i-4 j+k & \text { if } n \equiv 1(\bmod 4) \\
1-4 i+j+2 k & \text { if } n \equiv 2(\bmod 4) \\
-4+i+2 j+k & \text { if } n \equiv 3(\bmod 4)
\end{array},\right. \\
J Q_{n+2}^{(4)}-J Q_{n}^{(4)}-j Q_{n-1}^{(4)}=\left\{\begin{array}{cll}
-2 i+j+k & \text { if } n \equiv 0(\bmod 4) \\
-2+i+j & \text { if } n \equiv 1(\bmod 4) \\
1+i-2 k & \text { if } n \equiv 2(\bmod 4) \\
1-2 j+k & \text { if } n \equiv 3(\bmod 4)
\end{array},(n \geq 1) .\right.
\end{gathered}
$$

The following is a result for the sum of fourth-order Jacobsthal quaternions.

Theorem 3.4. Let $n \geq 0$ integer. Then,

$$
\sum_{s=0}^{n} J Q_{s}^{(4)}=\left\{\begin{array}{ccc}
J Q_{n+1}^{(4)}-(1+2 k) & \text { if } & n \equiv 0(\bmod 4) \\
J Q_{n+1}^{(4)}+(i-j-3 k) & \text { if } & n \equiv 1(\bmod 4) \\
J Q_{n+1}^{(4)}+(1-2 j-2 k) & \text { if } & n \equiv 2(\bmod 4) \\
J Q_{n+1}^{(4)}-(i+j+k) & \text { if } & n \equiv 3(\bmod 4)
\end{array} .\right.
$$

Proof. Using equality (1.12), we have

$$
\sum_{s=0}^{n} J_{s}^{(4)}=\left\{\begin{array}{ccc}
J_{n+1}^{(4)}-1 & \text { if } & n \equiv 0(\bmod 4) \\
J_{n+1}^{(4)} & \text { if } & n \equiv 1,3(\bmod 4) \\
J_{n+1}^{(4)}+1 & \text { if } & n \equiv 2(\bmod 4)
\end{array}\right.
$$


Furthermore, if $n \equiv 0(\bmod 4), \sum_{s=0}^{n} J_{s}^{(4)}=J_{n+1}^{(4)}-1, \sum_{s=0}^{n+1} J_{s}^{(4)}=J_{n+2}^{(4)}, \sum_{s=0}^{n+2} J_{s}^{(4)}=J_{n+3}^{(4)}+1$ and $\sum_{s=0}^{n+3} J_{s}^{(4)}=J_{n+4}^{(4)}$. Then,

$$
\begin{aligned}
\sum_{s=0}^{n} J Q_{s}^{(4)} & =\sum_{s=0}^{n} J_{s}^{(4)}+i \sum_{s=0}^{n} J_{s+1}^{(4)}+j \sum_{s=0}^{n} J_{s+2}^{(4)}+k \sum_{s=0}^{n} J_{s+3}^{(4)} \\
& =\sum_{s=0}^{n} J_{s}^{(4)}+i\left(\sum_{s=0}^{n+1} J_{s}^{(4)}\right)+j\left(\sum_{s=0}^{n+2} J_{s}^{(4)}-1\right)+k\left(\sum_{s=0}^{n+3} J_{s}^{(4)}-2\right) \\
& =\left(J_{n+1}^{(4)}-1\right)+i\left(J_{n+2}^{(4)}\right)+j\left(J_{n+3}^{(4)}\right)+k\left(J_{n+4}^{(4)}-2\right) \\
& =J Q_{n+1}^{(4)}-(1+2 k)
\end{aligned}
$$

If $n \equiv 1(\bmod 4)$, we have $\sum_{s=0}^{n+1} J_{s}^{(4)}=J_{n+2}^{(4)}+1, \sum_{s=0}^{n+2} J_{s}^{(4)}=J_{n+3}^{(4)}$ and $\sum_{s=0}^{n+3} J_{s}^{(4)}=J_{n+4}^{(4)}-1$, then $\sum_{s=0}^{n} J Q_{s}^{(4)}=J Q_{n+1}^{(4)}+(i-j-3 k)$. The proof is similar for the cases $n \equiv 2,3(\bmod 4)$. Thus, the proof is completed.

There are three well-known identities for Fibonacci numbers, namely, Catalan's, Cassini's, and d'Ocagne's identities. The proofs of these identities are based on Binet formulas. We can obtain these types of identities for fourth-order Jacobsthal quaternions using the Binet formulas derived above. We use the notation

$$
\begin{aligned}
H Q_{n}^{(4)}(a, b) & =\frac{A \omega_{1}^{n} \underline{\omega_{1}-B \omega_{2}^{n} \underline{\omega_{2}}}}{\omega_{1}-\omega_{2}} \\
& =\left\{\begin{array}{cll}
a+b i-a j-b k & \text { if } & n \equiv 0(\bmod 4) \\
b-a i-b j+a k & \text { if } & n \equiv 1(\bmod 4) \\
-a-b i+a j+b k & \text { if } & n \equiv 2(\bmod 4) \\
-b+a i+b j-a k & \text { if } & n \equiv 3(\bmod 4)
\end{array}\right.
\end{aligned}
$$

where $A=b-a \omega_{2}$ and $B=b-a \omega_{1}$, in which $\omega_{1}=1+\omega_{1} i-j-\omega_{1} k$ and $\omega_{2}=1+\omega_{2} i-j-\omega_{2} k$ are the complex conjugate quartic roots of unity (i.e. $\omega_{1}^{2}=\omega_{2}^{2}=-1$ ). Furthermore, note that for all $n \geq 0$ we have

$$
H Q_{n+2}^{(4)}(a, b)=-H Q_{n}^{(4)}(a, b),
$$

where $H Q_{0}^{(4)}(a, b)=a+b i-a j-b k$ and $H Q_{1}^{(4)}(a, b)=b-a i-b j+a k$.

The following theorem gives d'Ocagne's identities for fourth-order Jacobsthal quaternion.

Theorem 3.5. If $J Q_{n}^{(4)}=J_{n}^{(4)}+i J_{n+1}^{(4)}+j J_{n+2}^{(4)}+k J_{n+3}^{(4)}$ be the $n$-th fourth-order Jacobsthal quaternion. Then, for any integers $n$ and $m$, we have

$$
J Q_{m}^{(4)} J Q_{n+1}^{(4)}-J Q_{m+1}^{(3)} J Q_{n}^{(4)}=\frac{1}{5}\left\{\begin{array}{c}
2^{m} \alpha U Q_{n}^{(4)}-2^{n} U Q_{m}^{(4)} \alpha \\
-i\left(\omega_{1}^{m-n} \underline{\omega_{1} \omega_{2}}-\omega_{2}^{m-n} \underline{\omega_{2} \omega_{1}}\right)
\end{array}\right\}
$$

where $\alpha=1+2 i+4 j+8 k, \underline{\omega_{1}}=1+\omega_{1} i-j-\omega_{1} k, \underline{\omega_{2}}=1+\omega_{2} i-j-\omega_{2} k$ and $U Q_{n}^{(4)}=H Q_{n}^{(4)}(-1,-1)$.

Proof. Using the Binet formula for the fourth-order Jacobsthal quaternions and $V Q_{n}^{(4)}=H Q_{n}^{(4)}(1,-3)$ in (3.8) gives

$$
\begin{aligned}
& J Q_{m}^{(4)} J Q_{n+1}^{(4)}-J Q_{m+1}^{(4)} J Q_{n}^{(4)} \\
& =\frac{1}{25}\left(\begin{array}{c}
\left(2^{m} \alpha-V Q_{m}^{(4)}\right)\left(2^{n+1} \alpha-V Q_{n+1}^{(4)}\right) \\
-\left(2^{m+1} \alpha-V Q_{m+1}^{(4)}\right)\left(2^{n} \alpha-V Q_{n}^{(4)}\right)
\end{array}\right) \\
& =\frac{1}{25}\left(\begin{array}{c}
-2^{m} \alpha V Q_{n+1}^{(4)}-2^{n+1} V Q_{m} \alpha+2^{m+1} \alpha V Q_{n}^{(4)}+2^{n} V Q_{m+1}^{(4)} \alpha \\
+V Q_{m}^{(4)} V Q_{n+1}^{(4)}-V Q_{m+1}^{(4)} V Q_{n}^{(4)}
\end{array}\right) \\
& =\frac{1}{5}\left(2^{m} \alpha U Q_{n}^{(4)}-2^{n} U Q_{m}^{(4)} \alpha-i\left(\omega_{1}^{m-n} \underline{\omega_{1} \omega_{2}}-\omega_{2}^{m-n} \underline{\omega_{2} \omega_{1}}\right)\right),
\end{aligned}
$$

where $U Q_{n}^{(4)}=\frac{1}{5}\left(2 V Q_{n}^{(4)}-V Q_{n+1}^{(4)}\right)=H Q_{n}^{(4)}(1,-1)$.

Taking $m=n+1$ in this theorem and using the identity

$$
-i\left(\omega_{1} \underline{\omega_{1} \omega_{2}}-\omega_{2} \underline{\omega_{2} \omega_{1}}\right)=\underline{\omega_{1} \omega_{2}}+\underline{\omega_{2} \omega_{1}}=-4(1+j),
$$

we obtain Cassini's identities for fourth-order Jacobsthal quaternions.

Corollary 3.6. For any integer $n \geq 0$, we have

$$
\left(J Q_{n+1}^{(4)}\right)^{2}-J Q_{n+2}^{(3)} J Q_{n}^{(4)}=\frac{1}{5}\left(2^{n}\left(2 \alpha U Q_{n}^{(4)}-U Q_{n+1}^{(4)} \alpha\right)-4(1+j)\right) .
$$

We will give an example in which we check in a particular case the Cassini-like identity for fourth-order Jacobsthal quaternions. 
Example 3.7. Let $\left\{J Q_{s}^{(4)}: s=0,1,2,3\right\}$ be the fourth-order Jacobsthal quaternions such that $J Q_{0}^{(4)}=i+j+k, J Q_{1}^{(4)}=1+i+j+3 k$, $J Q_{2}^{(4)}=1+i+3 j+7 k$ and $J Q_{3}^{(4)}=1+3 i+7 j+13 k$. In this case,

$$
\begin{aligned}
\left(J Q_{1}^{(4)}\right)^{2}-J Q_{2}^{(4)} J Q_{0}^{(4)} & =(1+i+j+3 k)^{2}-(1+i+3 j+7 k)(i+j+k) \\
& =(-10+2 i+2 j+6 k)-(-11-3 i+7 j-k) \\
& =1+5 i-5 j+7 k \\
& =\frac{1}{5}\left(\left(2 \alpha U Q_{0}^{(4)}-U Q_{1}^{(4)} \alpha\right)-4(1+j)\right) .
\end{aligned}
$$

and

$$
\begin{aligned}
\left(J Q_{2}^{(4)}\right)^{2}-J Q_{3}^{(4)} J Q_{1}^{(4)} & =(1+i+3 j+7 k)^{2}-(1+3 i+7 j+13 k)(1+i+j+3 k) \\
& =(-58+2 i+6 j+14 k)-(-48+12 i+12 j+12 k) \\
& =-10-10 i-6 j+2 k \\
& =\frac{1}{5}\left(2\left(2 \alpha U Q_{1}^{(4)}-U Q_{2}^{(4)} \alpha\right)-4(1+j)\right) .
\end{aligned}
$$

\section{Conclusions}

In this work, some known identities of the sequence of Jacobsthal numbers have continued to be generalized with the use of the quaternion ring. The main motivation is based on the study of the non-commutative properties of the quaternions, and how we can solve friendly cases with sequences of recursive numbers. In particular, the ideas of finding rules of commutativity, matrix representation of quaternion sequences and their study in a wider class of rings, say in octonions or in any power associative ring.

\section{Acknowledgements}

The author also thanks the suggestions sent by the reviewer, which have improved the final version of this article.

\section{References}

[1] P. Barry, Triangle geometry and Jacobsthal numbers, Irish Math. Soc. Bulletin 51 (2003), 45-57.

[2] G. Cerda-Morales, Identities for Third Order Jacobsthal Quaternions, Adv. Appl. Clifford Algebras 27(2) (2017), 1043-1053.

[3] C.K. Cook and M.R. Bacon, Some identities for Jacobsthal and Jacobsthal-Lucas numbers satisfying higher order recurrence relations, Annales Mathematicae et Informaticae 41 (2013), 27-39.

[4] O. Deveci, E. Karaduman and G. Sağlam, The Jacobsthal sequences in finite groups, Bull. Iranian Math. Soc. 42(1) (2016), 79-89.

[5] S. Halici, On Fibonacci quaternions, Adv. Appl. Clifford Algebras 22 (2012), 321-327.

[6] S. Halici, On complex Fibonacci quaternions, Adv. Appl. Clifford Algebras 23 (2013), 105-112.

[7] A.F. Horadam, Complex Fibonacci numbers and Fibonacci quaternions, Am. Math. Month. 70 (1963), $289-291$.

[8] A.F. Horadam, Quaternion recurrence relations, Ulam Quarterly 2 (1993), 23-33.

[9] A.F. Horadam, Jacobsthal representation numbers, Fibonacci Quarterly 34 (1996), 40-54.

[10] M.R. Iyer, A note on Fibonacci quaternions, Fibonacci Quaterly 7(3) (1969), 225-229.

[11] D. Kalman, Generalized Fibonacci numbers by matrix methods, Fibonacci Quaterly 20(1) (1982), 73-76.

[12] F. Köken and D. Bozkurt, On the Jacobsthal numbers by matrix methods, Int. J. Contemp. Math. Sci. 3 (2008), 605-614.

[13] A. Szynal-Liana and I. Włoch, A Note on Jacobsthal Quaternions, Adv. Appl. Clifford Algebras 26 (2016), 441-447.

[14] F. Torunbalci Aydin and S.Yüce, A new approach to Jacobsthal quaternions, FILOMAT 31 (2017), 5567-5579. 